\title{
Shared mental healthcare and somatization: changes in patient symptoms and disability
}

\author{
John M. Haggarty ${ }^{1,2,3,4}$, Brian P. O'Connor ${ }^{5}$, Jeremy B. Mozzon ${ }^{6,7,8}$ and S. Kathleen Bailey ${ }^{9,10}$ \\ ${ }^{1}$ Medical Director, Mental Health Services, St. Joseph's Health Centre, Thunder Bay, Ontario, Canada \\ ${ }^{2}$ Director, Shared Mental Health Care, Fort William Clinic, Thunder Bay, Ontario, Canada \\ ${ }^{3}$ Professor, Department of Psychiatry, Northern Ontario School of Medicine, Thunder Bay, Ontario, Canada \\ ${ }^{4}$ Adjunct Professor, Department of Psychology, Lakehead University, Thunder Bay, Ontario, Canada \\ ${ }^{5}$ Professor, Department of Psychology, University of British Columbia Okanagan, Kelowna, British Columbia, Canada \\ ${ }^{6}$ Family Physician, NorWest Community Health Centre, Thunder Bay, Ontario, Canada \\ ${ }^{7}$ Lecturer, Department of Family Medicine, Dalhousie University, Truro, Nova Scotia, Canada \\ ${ }^{8}$ Lecturer/Assistant Professor, Northern Ontario School of Medicine, Thunder Bay, Ontario, Canada \\ ${ }^{9}$ Researcher, Mental Health Outpatients Services, St. Joseph's Health Centre, Thunder Bay, Ontario, Canada \\ ${ }^{10}$ Student, Department of Psychology, Lakehead University, Thunder Bay, Ontario, Canada
}

\begin{abstract}
Aim: To describe the symptoms and functional changes in patients with high levels of somatization who were referred to an outpatient, multidisciplinary, shared mental healthcare $(\mathrm{SMHC})$ service that primarily offered cognitive behavioural therapy. Second, we wished to compare the levels of somatization in this outpatient clinical sample with previously published community norms. Background: Somatization is common in primary care, and it can lead to significant impairment, disproportionate resource use, and poses a challenge for management. Methods: All the patients $(18+$ years, $n=508)$ who attended three or more treatment sessions in SMHC primary care over a seven-year period were eligible for inclusion to this pre-post study. Self-report measures included the Patient Health Questionnaire's somatic symptom severity scale (PHQ-15) and the World Health Organization Disability Assessment Schedule (WHODAS II). Normative comparisons were used to assess the degree of symptoms and functional changes. Findings: Clinically significant levels of somatization before treatment were common $(n=138,27.2 \%)$ and were associated with a significant reduction in somatic symptom severity $(41.3 \%$ reduction; $P<0.001)$ and disability $(44 \%$ reduction; $P<0.001)$ after treatment. Patients' levels of somatic symptom severity and disability approached but did not quite reach the community sample norms following treatment. Multidisciplinary short-term SMHC was associated with significant improvement in patient symptoms and disability, and shows promise as an effective treatment for patients with high levels of somatization. Including a control group would allow more confidence regarding the conclusions about the effectiveness of SMHC for patients impaired by somatization.
\end{abstract}

Key words: collaborative care; PHQ; primary care; somatization

Received 5 January 2015; revised 9 July 2015; accepted 19 July 2015; first published online 27 August 2015

\section{Introduction}

Physical symptoms, regardless of aetiology, can be intensely distressing and disabling. Patients with

Correspondence to: S. Kathleen Bailey, MA, PhD, Department of Psychology, Lakehead University, 955 Oliver Road, Thunder Bay, ON P7B 5E1, Canada. Email: skbailey@lakeheadu.ca

(C) Cambridge University Press 2015 high levels of somatization often present to primary care, with some studies suggesting a prevalence of 10-15\% (Fink et al., 1999; de Waal et al., 2004). Somatizing patients use a greater than average amount of healthcare resources, are often dissatisfied with their care (Jackson and Kroenke, 2008), and can be a source of frustration for the physicians responsible for their care (Lin et al., 1991; 
Hahn et al., 1996). Impairment caused by severe somatic symptoms has been found to be similar in effect to that of depressive and anxiety disorders (de Waal et al., 2004).

Despite its prevalence, somatization can be difficult to diagnose. Patients and physicians may be reluctant to accept a psychiatric diagnosis for their experience of physical symptoms (Mai, 2004). Somatization is often co-morbid with medical and other psychiatric disorders, such as depression, anxiety, and substance use disorder (Mai, 2004; Muller et al., 2008). However, few patients are referred to mental healthcare services specifically for the management of their somatization symptoms (de Jonge et al., 2001; Muller et al., 2008).

Experts recommend a combination of behavioural and cognitive interventions for excessive somatizing, such as providing short, frequent, and structured visits; limiting medical investigations and referrals; treating co-morbid medical and psychiatric conditions; pharmacotherapy; and psychotherapy (Mai, 2004; Cucciare and Lillis, 2009). Cognitive behavioural therapy (CBT) was found to be one of the most effective and widely validated treatment modalities for highly somatizing patients based on available literature (Kroenke and Swindle, 2000; Smith et al., 2003; Mai, 2004; Kroenke, 2007; Sumathipala, 2007). A systematic review by Kroenke (2007) found only four randomized controlled trials focussing specifically on somatoform disorder, with nine others investigating treatments for related disorders. The author concluded that CBT was the 'best established treatment' (Kroenke, 2007: 414). Sumathipala's (2007) review of CBT and somatoform disorders in the same year concluded that CBT was helpful in reducing physical symptoms, psychological distress, and disability. Few studies on the treatment of highly somatizing patients with CBT have been conducted in primary-care settings or co-located mental healthcare services.

Shared mental healthcare (SMHC) involves the delivery of outpatient mental healthcare services in the patient's primary healthcare setting using a collaborative, inter- and intra-professional team approach. SMHC was developed to improve accessibility to mental healthcare services and improve communication between psychiatry and family physicians (Kates et al., 1997; Farrar et al., 2001). Family physicians involved in collaborative care report greater knowledge, skills, and satisfaction in managing patients with psychiatric disorders (Kisely et al., 2006). SMHC practice is described in greater detail elsewhere (Haggarty et al., 2008b).

This study is a continuation of the ongoing work by this research team to examine the effectiveness of the SMHC model in the treatment of common mental disorders (Haggarty et al., 2008a; 2008b; 2012). The purpose of this study was to describe symptom improvement and change in function in patients with high levels of somatization, who were referred from primary care to an outpatient SMHC service, and to compare levels of somatization in this primary-care-referred clinical population with community norms.

\section{Methods}

Patients in a primary-care practice were followedup over a seven-year period. Clinical data were obtained as part of the clinic's regular intake and assessment procedures. The potential use of clinical data for research and programme monitoring purposes was explained to every patient on their first visit to the clinic, and all patients were given the option of refusing to participate or to withdraw at any time point without consequence. Patients were explained the risks and benefits of participating in the research programme as well as the anonymization procedure. Clinical measures [World Health Organization Disability Assessment Schedule (WHODAS II), Patient Health Questionnaire (PHQ)] were repeated after three or more SMHC treatment sessions. An Institutional Review Board approved this study. All the patients included in the study provided their free and informed consent.

The clinical setting was a geographically isolated but urban SMHC service co-located within a primary-care group practice with a roster of $\sim 18,000$ patients. The mental healthcare team included two full-time mental health counsellors (registered social workers) and a psychiatrist who provided one half-day of clinic hours every two weeks and phone support for counsellors and family physicians as required. Referrals to SMHC were made by family physicians within the clinic. The standard procedure was to establish telephone contact with the patient within $48 \mathrm{~h}$ of the referral. The average wait time for counselling was about 
two to three weeks and, when indicated, three to six weeks for psychiatric assessment.

\section{Clinical intervention}

Members of the mental healthcare team were trained and experienced in manualized forms of CBT, psycho-education, and supportive counselling. Although counsellors independently chose and applied interventions appropriate to the client's need, CBT was the preferred intervention. Clinical case reviews with the psychiatrist occurred as required by either the primary-care physician or the counsellor during bi-weekly clinic hours and by phone. The target counselling duration was six to eight treatment sessions per course of care. Psychiatric services were predominantly consultative, with a focus on medication management.

\section{Sample characteristics}

There were 2859 patients referred to SMHC over the seven-year study period (2002-2009). Approximately one person per year opted not to participate in the study. Pre-treatment assessment measures were obtained from 1529 cases $(53.4 \%)$. To accurately assess the benefit of a course of SMHC counselling, patients were required to attend at least three treatment sessions before post-treatment measures were requested. A total of 957 patients were seen for three or more sessions, and post-treatment measures were obtained from 508 patients $(53.1 \%)$. The average age of patients referred to SMHC services was 38.0 $(\mathrm{SD}=15.1)$ and $70.2 \%$ were female.

\section{Clinical assessment measures}

Patients completed the full PHQ and the WHODAS II before initial assessment and at the end of the treatment visit, if they attended more than three treatment sessions. PHQ items correspond to DSM-IV diagnostic criteria and were designed to assist with the identification and diagnosis of the most common mental disorders seen in primary care. The PHQ has shown to be accurate as a diagnostic tool, to demonstrate good sensitivity and specificity, and to correlate well with mental healthcare practitioners' diagnoses and objective measures of functional impairment (Spitzer et al., 1999).
The Patient Health Questionnaire somatic symptom severity scale (PHQ-15) is a subset of the full PHQ. The PHQ-15 is a validated screening tool for assessing the severity of somatic symptoms (Kroenke et al., 2002; Interian et al., 2006). Scores on the PHQ-15 range from 0 to 30 , with higher scores indicating more severe somatic symptoms. PHQ-15 scores $\geqslant 15$ are predictive of clinically significant or high levels of somatization in primary care.

We used the English version of the WHODAS II to assess disability change following treatment. The WHODAS II is a 12-item screening questionnaire that quantifies the disability that a person experiences in a variety of daily settings as a result of physical and mental health symptoms. Scores range from 0 to 48, and higher scores are associated with greater disability. This measure has been extensively validated and is available in multiple languages (Federici and Meloni, 2010). Patients also provided responses to the supplementary health/interference WHODAS II items that asked them to rate their overall health in the past 30 days (0-4), rate of the overall degree of interference of the problems in their lives (0-4), indicate the number of days with difficulty in the last 30 days, indicate the number of days with total incapacity in the last 30 days, and indicate the number of days of reduced activity in the last 30 days.

\section{Statistical analyses}

Mean entry and exit scores were compared using paired $t$-tests. Only patients with pre- and post-intervention data were included in the primary analyses.

To minimize bias and address the high rates of patients lost to follow-up, intention to treat (ITT) analyses were performed, which included all 957 patients who completed three or more sessions of SMHC. For those patients without postintervention measures, entry data were carried forward and used as exit data. All the analyses were performed using SPSS version 23.

We also used normative comparison procedures (Kendall et al., 1999) to examine the effectiveness of the treatment. We sought to determine whether the post-treatment means of our sample differed from community norms and whether the posttreatment means were significantly $<1$ SD above 
the community norms. Treatment and community sample groups were considered 'clinically equivalent' when the post-treatment mean was not significantly different from the community norm and when the post-treatment mean was significantly $<1 \mathrm{SD}$ above the community norm.

\section{Results}

\section{Whole-sample characteristics}

For patients completing three or more treatment sessions in SMHC, the average number of sessions was $7.3(\mathrm{SD}=3.7)$. The mean number of days between entry and exit was $166(\mathrm{SD}=113)$. The percentages of patients meeting the PHQ diagnostic thresholds (15 or greater) were as follows: $38.7 \%$ for major depressive syndrome, $20.9 \%$ for panic syndrome, $29.7 \%$ for other anxiety disorder, and $23.5 \%$ met the threshold for alcohol abuse. Scores indicating clinically significant levels of somatic complaints were present for $25.8 \%$ of the sample.

Most patients were referred to SMHC for depressed mood $(62.6 \%)$, anxiety symptoms $(42.2 \%)$, panic symptoms $(15.8 \%)$, sleep disturbance $(25.1 \%)$, or a combination of these. Only $5 \%$ of all patients were referred for excessive somatic symptoms, and $9 \%$ were referred for chronic pain. Of the patients with high levels of somatic complaints (PHQ-15 scores of 15 or greater), $8.1 \%$ were referred for excessive somatic symptoms and $15.9 \%$ were referred for chronic pain.

Post-treatment measures were not obtained for the following reasons: $64 \%$ withdrew before the final treatment session, $19 \%$ completed treatment but exit data were not obtained, $11 \%$ were referred or transferred to another service, and $7 \%$ for miscellaneous reasons. The baseline scores for patients who did and who did not complete the post-treatment measures were not statistically different.

\section{Characteristics of high- and low-somatization patients}

Only patients who completed three or more sessions of SMHC with both entry and exit data were included in the analyses reported below. Of the 508 patients with complete data, 138 patients (27.2\%) had PHQ-15 scores indicating clinically significant levels of somatization, as defined by a PHQ-15 score of 15 or more. The remaining participants $(72.8 \%)$ had PHQ-15 scores indicating non-significant levels of somatization.

The patients in the high-somatization group were on average two and a half years younger $(P=0.001)$, were more likely to be female ( 87.1 versus $74.5 \%$ ), and had more sessions to the emergency room in the past year than those who did not meet the somatization symptom threshold. The co-morbid psychiatric disorders suggested by PHQ diagnostic thresholds in patients with high and low levels of somatization are summarized in Table 1. The high-somatization patients were more likely to suffer from alcohol abuse, major depressive syndrome, panic, other anxiety disorders, and binge-eating disorder.

\section{Entry-versus-exit comparisons}

The entry and exit means, standard deviations, and effect sizes for the high- and low-somatization groups are provided in Table 2. The average

Table 1 Co-morbid disorders for patients above and below the threshold for somatization (PHQ-15 $\geqslant 15)$

\begin{tabular}{llrr}
\hline & $\begin{array}{l}\text { Percentage of highly somatic } \\
\text { patients with disorder }\end{array}$ & $\begin{array}{l}\text { Percentage of less somatic } \\
\text { patients with disorder }\end{array}$ \\
\hline Alcohol abuse & 31.8 & 19.5 & 0.001 \\
Binge-eating disorder & 12.3 & 7.6 & 0.013 \\
Bulimia nervosa & 3.3 & 1.6 & 0.066 \\
Other anxiety disorder & 59.9 & 19.8 & 0.001 \\
Panic syndrome & 36.1 & 14.6 & 0.001 \\
Major depression syndrome & 69.5 & 27.4 & 0.001 \\
Other depressive disorder & 7.3 & 11.2 & 0.052 \\
\hline
\end{tabular}

PHQ-15 = Patient Health Questionnaire's somatic symptom severity scale. 
Table 2 Means, standard deviations, and effect sizes for patients completing both baseline and exit measures

\begin{tabular}{|c|c|c|c|c|c|}
\hline & Entry mean & Entry SD & Exit mean & Exit SD & $d$ \\
\hline \multicolumn{6}{|l|}{ High-somatization patients } \\
\hline PHQ-15 & 18.1 & 2.5 & 10.6 & 5.2 & 1.80 \\
\hline WHODAS II & 36.5 & 17.3 & 20.4 & 17.2 & 0.92 \\
\hline Overall health in the past 30 days $(0-4)$ & 2.6 & 0.9 & 3.3 & 0.9 & 0.88 \\
\hline Overall interference with life (0-4) & 3.3 & 1.0 & 2.1 & 1.0 & 1.21 \\
\hline Number of days with difficulty in the last 30 days & 21.5 & 8.9 & 12.0 & 10.4 & 0.99 \\
\hline Number of days with total incapacity in the last 30 days & 9.3 & 10.6 & 3.5 & 7.0 & 0.65 \\
\hline Number of days of reduced activity in the last 30 days & 13.4 & 10.1 & 6.8 & 8.8 & 0.70 \\
\hline \multicolumn{6}{|l|}{ Low-somatization patients } \\
\hline PHQ-15 & 8.8 & 3.5 & 5.3 & 3.7 & 0.98 \\
\hline WHODAS II & 20.8 & 15.3 & 10.2 & 12.4 & 0.77 \\
\hline Overall health in the past 30 days $(0-4)$ & 2.1 & 0.8 & 2.7 & 0.8 & 0.79 \\
\hline Overall interference with life (0-4) & 2.5 & 1.0 & 1.7 & 0.8 & 0.93 \\
\hline Number of days with difficulty in the last 30 days & 16.6 & 10.2 & 7.5 & 10.1 & 0.90 \\
\hline Number of days with total incapacity in the last 30 days & 4.0 & 7.6 & 1.4 & 4.7 & 0.40 \\
\hline Number of days of reduced activity in the last 30 days & 8.3 & 9.4 & 3.2 & 6.9 & 0.61 \\
\hline
\end{tabular}

PHQ-15 = Patient Health Questionnaire's somatic symptom severity scale; WHODAS II = World Health Organization Disability Assessment Schedule.

All means in the same row are significantly different at the 0.001 level. The $d$ values are effect sizes, representing the differences between means in standard deviation units.

PHQ-15 score for patients with clinically significant somatization decreased from 18.08 $(\mathrm{SD}=2.54)$ to $10.61(\mathrm{SD}=5.20)$ after treatment, representing a significant $(41.3 \%, \quad t=16.6$, $P<0.0001, d=1.8$ ) reduction in somatic symptom severity following three or more sessions of counselling with SMHC. Patients who initially had nonclinical levels of somatization also demonstrated a significant decrease $(39.6 \%)$ in their somatizing symptoms following treatment with SMHC $(t=18.7, P>0.0001)$. The pre-treatment mean was $8.81(\mathrm{SD}=3.45)$, the post-treatment mean was $5.32(\mathrm{SD}=3.67)$, and the effect size was $d=0.98$. Both the entry-versus-exit findings for the PHQ-15 were also significant for both males and females.

Patients with high levels of somatic symptoms showed statistically significant entry-to-exit SMHC treatment reductions in mean WHODAS II scores $(t=11.2, P<0.0001)$. The entry and exit means were $36.53(\mathrm{SD}=17.34)$ and $20.42(\mathrm{SD}=17.15)$, respectively, indicating a $44.1 \%$ decrease $(d=0.92)$ in self-reported disability related to mental illness following SMHC intervention. Patients with lower levels of somatization experienced a $51.2 \%(t=16.1, P<0.0001)$ decrease in self-reported disability. The mean decreased from $20.83(\mathrm{SD}=15.33)$ on entry to $10.16(\mathrm{SD}=12.35)$ after intervention, and the effect size was $d=0.77$. Both the entry-versus-exit findings for the WHODAS II were also significant for both males and females.

The findings for the supplementary WHODAS II items are also reported in Table 2. All the entry-versus-exit comparisons were significantly different, for both the high- and low-somatization groups. Self-ratings of health improved, there was less interference of the problems in their lives, there were fewer days with difficulty in the last 30 days, fewer days with total incapacity in the last 30 days, and fewer days of reduced activity in the last 30 days.

ITT analyses were conducted for all 957 patients who completed three or more sessions of SMHC. In these analyses, the scores at entry for those patients without post-intervention measures were carried forward and repeated (used) as their exit scores. Although the effect sizes were predictably weaker, the pattern of significant findings remained unchanged. The entry-versus-exit reduction in PHQ-15 scores was significant for the high-somatization group $(t=11.8, P<0.0001$, $d=0.81)$ and for the low-somatization group $(t=15.9, \quad P<0.0001, \quad d=0.51)$. Similarly, the entry-versus-exit reduction in WHODAS II scores was significant for the high-somatization group

Primary Health Care Research \& Development 2016; 17: 277-286 
$(t=9.3, P<0.001, d=0.81)$ and for the lowsomatization group $(t=14.0, P<0.0001, d=0.37)$.

\section{Moderators of treatment}

Multiple regression analyses were conducted to determine whether the high- and low-somatization groups changed at the same rate across time. Scores at entry were entered first followed by the somatization group (high/low) for the prediction of scores upon exit (identical results were obtained when analysis of covariance was used instead of regression). The $R^{2}$ change for the addition of the somatization group was not significant for PHQ-15 $\left(R^{2}=0.003, F=2.73, P=0.099\right.$, standardized $\beta=0.09)$. However, the $R^{2}$ change for the addition of the somatization group was significant for the WHODAS II $\left(R^{2}=0.005, F=3.92, P=0.048\right.$, standardized $\beta=0.08)$. The size of the effect was very small, as revealed by near-parallel lines for the two somatization groups (see Figure 2).

Additional analyses focussed on variables that may predict variation in scores at entry and variation in responses to treatment. Exit-versus-entry difference scores were used to quantify the magnitude of change. Women had higher PHQ-15 scores upon entry than men $(M=11.7, \mathrm{SD}=5.1$ for females, $M=9.7, \mathrm{SD}=5.1$ for males, $t=4.2$, $P<0.001, d=0.39$ ), and women's somatic symptoms changed more than men's from entry to exit $(M=-4.9, \mathrm{SD}=4.5$ for females, $M=-3.4$, $\mathrm{SD}=4.4$ for males, $t=4.0, P<0.001, d=0.33$ ). Women did not have higher WHODAS II scores upon entry than men $(M=25.1, \mathrm{SD}=17.4$ for females, $M=23.9, \mathrm{SD}=17.0$ for males, $t=0.7$,
$P=0.48, d=0.07)$, but women changed more than men from entry to exit $(M=-12.9, \mathrm{SD}=14.1$ for females, $M=-9.7, \mathrm{SD}=13.4$ for males, $t=2.3, P=0.02, d=0.24)$.

Pearson's correlations for other potential moderators are provided in Table 3 . Most of the correlations were modest, with the strongest associations occurring for entry scores, especially for the WHODAS II. Higher scores were associated with more hospital sessions, more emergency room sessions, and more crisis-centre calls at entry. The correlations between these variables and change over time were quite modest.

\section{Normative comparisons}

Kroenke et al. (2002) reported a PHQ-15 mean of $3.4(\mathrm{SD}=3.8)$ for a large, nationally representative sample of the general population. The community sample mean for the WHODAS II is 6.41 (SD = 11.42; S. Chatterji, 2006, personal communication). The results of the normative comparison analyses are depicted in Figures 1 and 2 ; they are easily summarized (to preserve space). The entry-to-exit reductions in symptoms are clearly visible for both measures. The high- and low-somatization groups had different elevations at entry and exit, but the lines are parallel and the slopes are the same for the two groups. The low-somatization group scored slightly above community norms at entry, and their scores dropped $<1$ SD above the community norms at exit, although not significantly $<1 \mathrm{SD}$ point. The high-somatization group scored well above the community norms at entry, and their means

Table 3 Pearson's correlations for potential moderators of status on entry and change over time

\begin{tabular}{|c|c|c|c|c|}
\hline & PHQ-15 entry & PHQ-15 change & WHODAS II entry & WHODAS II change \\
\hline Age & 0.01 & 0.08 & $0.13^{*}$ & 0.00 \\
\hline Number of treatment sessions & 0.10 & 0.07 & $0.17^{*}$ & -0.06 \\
\hline Number of days in SMHC & $0.12^{*}$ & 0.10 & $0.12 *$ & 0.00 \\
\hline Number of hospitalizations in the past year & 0.07 & 0.09 & $0.16^{*}$ & 0.01 \\
\hline Number of ER visits in the past year & $0.20 *$ & 0.01 & $0.13^{*}$ & 0.09 \\
\hline Number of ER visits for mental health & $0.17^{*}$ & $-0.14^{*}$ & $0.14^{*}$ & -0.09 \\
\hline Number of crisis-centre calls in past year & 0.10 & -0.01 & $0.14^{*}$ & 0.05 \\
\hline
\end{tabular}

PHQ-15 = Patient Health Questionnaire's somatic symptom severity scale; WHODAS II = World Health Organization Disability Assessment Schedule; $\mathrm{SMHC}=$ shared mental healthcare; $\mathrm{ER}=$ emergency room.

'Change' = exit - entry difference scores.

${ }^{*} P<0.05$.

Primary Health Care Research \& Development 2016; 17: 277-286 


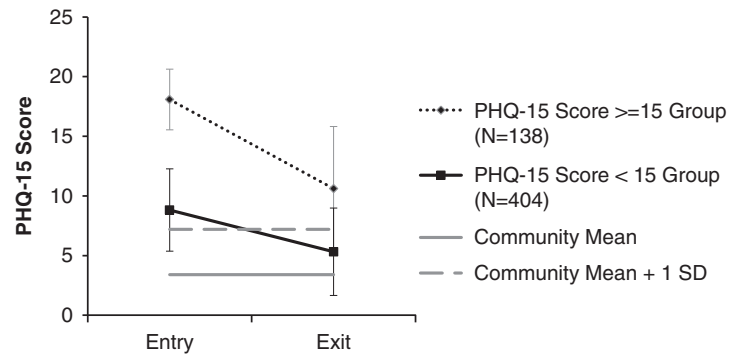

Figure 1 Entry-versus-exit normative comparisons for PHQ-15 means before and after treatment with the shared mental healthcare service. The solid grey line represents the PHQ-15 community mean, whereas the dotted grey line represents 1 SD above the mean. Scores within 1 SD of the community mean are considered 'normal'. The error bars represent $1 \mathrm{SD}$. PHQ-15 = Patient Health Questionnaire's somatic symptom severity scale.

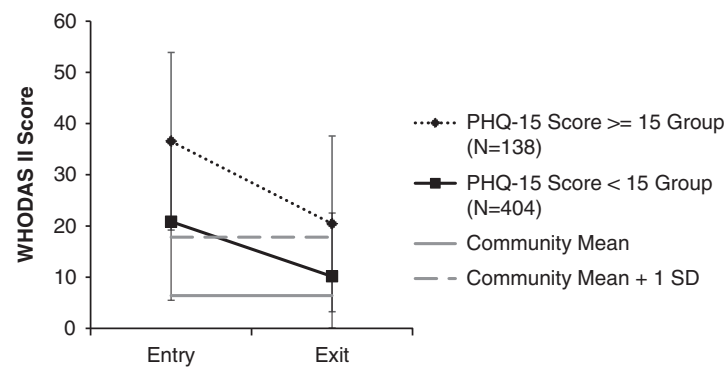

Figure 2 Entry-versus-exit normative comparisons for WHODAS II means before and after treatment with the shared mental healthcare service. The solid grey line represents the PHQ-15 community mean, whereas the dotted grey line represents $1 \mathrm{SD}$ above the mean. Scores within 1 SD of the community mean are considered 'normal'. The error bars represent 1 SD. WHODAS II = World Health Organization Disability Assessment Schedule; PHQ-15 = Patient Health Questionnaire's somatic symptom severity scale.

approached, but remained slightly above, community norms after treatment. Clinical equivalence was, thus, approached but not obtained.

\section{Discussion}

The multidisciplinary, short-term shared care intervention was an effective treatment for patients with clinically significant somatic symptoms. This group of patients with high levels of somatic complaints demonstrated significant improvements in physical symptom severity and self-reported disability. The effect sizes for the entry-versus-exit changes were substantial, and exit somatic symptom and disability scores approached community norms. These findings are consistent with the conclusions of systematic reviews evaluating the effectiveness of intervention for patients with somatic symptom disorders (Smith et al., 2003; Kroenke, 2007; Sumathipala, 2007).

It is noteworthy that most of the patients with clinically significant levels of somatization were not referred for somatic symptoms. Moreover, many patients with high levels of somatization had co-morbid psychiatric disorders. These results suggest that the typical patient with multiple somatic complaints seen by primary-care providers may be a 'somatically active' patient with depression and anxiety. PHQ-15 scores may be one measure that reflects the degree of distress caused by physical symptoms, which could explain the responsiveness of patients to brief psychological interventions. Somatic symptom severity may have improved directly from intervention with SMHC, secondary to treatment of co-morbid psychiatric illness with SMHC, and/or other factors, although determining their relative impact on symptom improvement is beyond the scope of this study.

The statistical effect sizes for the entry-versusexit comparisons were almost all in the 'large' range, based on conventional interpretations of $d$ values. The statistical effect sizes were on par with the stronger effect sizes that are reported in typical treatment outcome studies. Although there are no conventions for how much change in our measures is required for clinical significance, the normative comparison results help place our findings in a more practical perspective. After treatment, the high-somatization group means approached but remained just slightly $>1$ SD above the community norm. The low-somatization group means went from $>1$ SD above community norm values upon entry to slightly $<1$ SD mark after treatment. The post-treatment means for both groups remained significantly above community norms. The high-somatization patients thus improved notably, but they still had ways to go. Further steps for improvement may be more challenging than the first few steps, and they may require longer treatments.

Or perhaps not, given the findings depicted in Figures 1 and 2. The downward slopes in somatic symptoms and disability were essentially the same 
for the high- and low-somatization groups. Perhaps more importantly, the levels that were attained by the high-somatization group after treatment were very similar to the initial levels of the low-somatization group, which eventually dropped down much closer to the community norms upon exit. The lines in the figures, thus, suggest that therapy was only half done for the high-somatization group and that further change is possible.

The ITT analyses, which included all the patients who attended three or more sessions of SMHC, indicated statistically significant decreases in somatic symptoms and self-reported disability scores. However, these improvements were predictably more modest compared with the non-ITT analyses.

More generally, the results of these analyses suggest that CBT and personalized therapy provided by SMHC are viable forms of treatment for patients with multiple physical complaints, especially if there are co-morbid mood and anxiety symptoms. Treating somatically active patients in SMHC may have some secondary benefits, including potentially decreasing the need for other treatment referrals, unnecessary expensive and/or burdensome investigations, emergency room sessions, and medication prescriptions for symptom management. Several studies involving treatment of distressing somatic symptoms with CBT/counselling have demonstrated decreased healthcare utilization (Sumathipala et al., 2000; Martin et al., 2007). Further studies are required to assess the cost-benefit of the treatment of patients with clinically significant somatic symptoms in an SMHC setting, the optimal intervention approach, and to determine the direct and indirect effect of this model on somatic symptom severity and functional impairment.

\section{Moderators of treatment effects}

We examined several possible moderators of the SMHC intervention. A number of statistically significant effects emerged, but they were generally modest. Although symptoms were reduced in both men and women from entry to exit, women reported more somatic symptoms than men upon entry and they subsequently changed more compared with men. Men and women, thus, apparently benefit from SMHC, and women benefit slightly more than men. Age was not a significant predictor of change or of initial status.

Primary Health Care Research \& Development 2016; 17: 277-286
Upon entry, somatic symptom and disability scores were mildly associated with numbers of hospitalizations, emergency room visits, and crisiscentre calls in the past year. However, the associations between these variables and the degree of change during the SMHC programme were negligible. Patients who eventually had more treatment visits than other patients tended to score higher on disability upon entry, but the pattern of change over time was not meaningfully associated with visitation frequencies. The identification of treatment moderator variables that have notably stronger effects than our moderator variables is a challenge for further research.

\section{Limitations}

All the patients were offered treatment with the SMHC service, thus minimizing potential selection biases. However, causal inferences regarding the association between the SMHC intervention and changes in somatization symptoms and disability cannot be made from the present pre-post research design. The findings are promising, but merely suggestive. Given the absence of a control group, we conducted normative comparisons using previously published community data instead. Normative comparisons provide a more conservative, different, and highly relevant perspective on the research question in therapy outcome studies. In a traditional design where a control group is available, the focus is on the relative improvement displayed by the treatment group in comparison with the no-treatment group. In normative comparisons, the contrast is not with untreated patients but with community norms. The research question - did the treated group 'return to normal?' - is thus more stringent and more practically meaningful than is typically the case with control group research. In our case, the patients experienced significantly fewer symptoms after treatment than they did before treatment, they approached community norms, and they were almost but not quite 'returned to normal'.

Another limitation was the relatively low percentage of patients with completed exit measures. However, the patients who did not complete exit measures were not significantly different at baseline from those for who did complete the exit measures. ITT analyses were also performed to 
minimize bias and conservatively address loss to follow-up. The effect sizes from the ITT analyses were naturally smaller, but the patterns in the data remained unchanged.

Finally, longer-term follow-ups would be desirable for further research. Our exit measures were taken at the end of the last encounter with SMHC. A previous study (Haggarty et al., 2008a) did show that patients receiving brief psychotherapy and psychiatric consultation in the same SMHC service had sustained improvements in major depressive, anxiety, and somatization symptoms and that these improvements were maintained for three to six months following treatment.

\section{Conclusions}

Patients with clinically significant levels of somatic complaints can be treated with short-term counselling in a co-located primary-care setting and they can experience moderate-to-large improvements in physical symptoms and levels of disability. Further work including trials with control groups, longer-term follow-ups, and cost-benefit analyses will help refine the use of CBT and personalized counselling for patients with clinically significant levels of somatization.

\section{Acknowledgements}

This research was funded in part from the AHSC AFP Innovation Fund, awarded through the Northern Ontario School of Medicine. The authors wish to thank St. Joseph's Care Group and Vice President Janet Sillman and Dr. Lois Hutchinson for their support. To the counsellors and physicians of the Fort William Clinic for supporting the SMHC model, and administrative support throughout by Shannon Frizzell.

\section{Financial Support}

This research was funded in part by the Academic Health Sciences Centre Alternate Funding Plan Innovation Fund, awarded through the Northern Ontario School of Medicine.

\section{Conflicts of Interest}

None.

\section{Ethical Standards}

The authors assert that all the procedures contributing to their work comply with the ethical standards of the relevant national and institutional guidelines on human experimentation (Thunder Bay Regional Health Sciences Centre; St. Joseph's Care Group) and with the Helsinki Declaration of 1975, as revised in 2008.

\section{References}

Cucciare, M.A. and Lillis, J. 2009: Somatization in primary care. In James, L.C. and O'Donohue, W.T., editors, The primary care toolkit: practical resources for the integrated behaviour care provider. Chapter 21. New York, NY: Springer Science and Business Media, 307-22.

de Jonge, P., Huyse, F., Herzog, T., Lobo, A., Malt, U., Opmeer, B., Kuiper, B. and Krabbendam, A. 2001: Referral pattern of neurological patients to psychiatric ConsultationLiaison Services in 33 European hospitals. General Hospital Psychiatry 23, 152-57.

de Waal, M., Arnold, I., Eekhof, J. and van Hemert, A. 2004: Somatoform disorders in general practice: prevalence, functional impairment and comorbidity with anxiety and depressive disorders. British Journal of Psychiatry 184, 470-76.

Farrar, S., Kates, N., Crustolo, A. and Nikolaou, L. 2001: Integrated model for mental health care: are health care providers satisfied with it. Canadian Family Physician 47, 2483-488.

Federici, S. and Meloni, F. 2010: WHODAS II: disability selfevaluation in the ICF conceptual frame. In Stone, J. and Blouin, M., editors, International encyclopedia of rehabilitation. State University of New York, Buffalo, NY: CIRRIE. Retrieved 14 August 2015 from http://cirrie.buffalo.edu/ encyclopedia/en/article/299/.

Fink, P., Sorensen, L., Engberg, M., Holm, M. and Munk-Jorgensen, P. 1999: Somatization in primary care: prevalence, health care utilization, and general practitioner recognition. Psychosomatics 40, 330-38.

Haggarty, J., Jarva, J., Cernovsky, Z., Karioja, K. and Martin, L. 2012: Wait time impact of co-located primary care mental health services: the effect of adding collaborative care in Northern Ontario. Canadian Journal of Psychiatry 57, 29-33.

Haggarty, J., Klein, R., Chaudhuri, B., Boudreau, D. and McKinnon, T. 2008a: After shared care: patients' symptoms and functioning 3 to 6 months following care at a rural shared mental health care clinic. Canadian Journal of Community Mental Health 27, 47-54.

Haggarty, J., O'Connor, B., Dubois, S., Blackadar, A., McKinnon, T., Boudreau, D. and Haslam, D. 2008b: A pilot study of a Canadian shared mental health care programme: changes in patient symptoms and disability. Primary Care and Community Psychiatry 13, 27-35. 
Hahn, S., Kroenke, K., Spitzer, R., Brody, D., Williams, J., Linzer, M. and deGruy, F. 1996: The difficult patient in primary care: prevalence, psychopathology and impairment. Journal of General Internal Medicine 11, 1-8.

Interian, A., Allen, L., Gara, M., Escobar, J. and Diaz-Martinez, A. 2006: Somatic complaints in primary care: further examining the validity of the Patient Health Questionnaire (PHQ-15). Psychosomatics 47, 392-98.

Jackson, J. and Kroenke, K. 2008: Prevalence, impact, and prognosis of multisomatoform disorder in primary care: a 5-year follow-up study. Psychosomatic Medicine 70, 430-34.

Kates, N., Craven, M., Bishop, J., Clinton, T., Kraftcheck, D., LeClair, K., Leverette, J., Nash, L. and Turner, T. 1997: Shared mental health care in Canada. Canadian Journal of Psychiatry 42 (Suppl), 12pp.

Kendall, P., Marrs-Garcia, A., Nath, S. and Sheldrick, R. 1999: Normative comparisons for the evaluation of clinical significance. Journal of Consulting and Clinical Psychology 67, 285-99.

Kisely, S., Duerden, D., Shaddick, S. and Jayabarathan, A. 2006: Collaboration between primary and psychiatric services: does it help family physicians? Canadian Family Physician 52, 875-77.

Kroenke, K. 2007: Efficacy of treatment for somatoform disorders: a review of randomized controlled trials. Psychosomatic Medicine 69, 881-88.

Kroenke, K., Spitzer, R.L. and Williams, J.B.W. 2002: The PHQ-15: validity of a new measure for evaluating the severity of somatic symptoms. Psychosomatic Medicine 64, 258-66.

Kroenke, K. and Swindle, R. 2000: Cognitive-behavioral therapy for somatization and symptom syndromes: a critical review of controlled clinical trials. Psychotherapy and Psychosomatics 69, 205-15.
Lin, E.H., Katon, W., Von Korff, M., Bush, T., Lipscomb, P., Russo, J. and Wagner, E. 1991: Frustrating patients: physician and patient perspectives among distressed high users of medical services. Journal of General Internal Medicine 6, 241-46.

Mai, F. 2004: Somatization disorder: a practical review. Canadian Journal of Psychiatry 49, 652-62.

Martin, A., Rauh, E., Fichter, M. and Rief, W. 2007: A onesession treatment for patients suffering from medically unexplained symptoms in primary care: a randomized clinical trial. Psychosomatics 48, 294-303.

Muller, J.E., Wentzel, I., Nel, D.G. and Stein, D.J. 2008: Depression and anxiety in multisomatoform disorder: prevalence and clinical predictors in primary care. South African Medical Journal 98, 473-76.

Smith, R.C., Lein, C., Collins, C., Lyles, J.S., Given, B., Dwamena, F.C., Coffey, J., Hodges, A., Gardiner, J.C., Goddeeris, J. and Given, C.W. 2003: Treating patients with medically unexplained symptoms in primary care. Journal of General Internal Medicine 18, 478-89.

Spitzer, R.L., Kroenke, K. and Williams, J.B. 1999: Validation and utility of a self-report version of PRIME-MD: the PHQ primary care study. Primary care evaluation of mental disorders. Patient Health Questionnaire. Journal of the American Medical Association 282, 1737-744.

Sumathipala, A. 2007: What is the evidence for the efficacy of treatments for somatoform disorders? A critical review of previous intervention studies. Psychosomatic Medicine 69, 889-900.

Sumathipala, A., Hewege, S., Hanwella, R. and Mann, A.H. 2000: Randomized controlled trial of cognitive behaviour therapy for repeated consultations for medically unexplained complaints: a feasibility study in Sri Lanka. Psychological Medicine 30, 747-57. 